Note

\title{
Enantiomeric Determination of DOPA in Dietary Supplements Containing Mucuna pruriens by Liquid Chromatography/Mass Spectrometry
}

(Received March 27, 2013)

\author{
Takashi Hasegawa*, Kazunaga Takahashi, Tomohide Fukiwake, Masaaki Saijo and Yuji Moтокi \\ Chiba Prefectural Institute of Public Health: 666-2 Nitona-cho, Chuo-ku, Chiba 260-8715, Japan; \\ * Corresponding author
}

\begin{abstract}
We developed a simple and rapid liquid chromatography/mass spectrometry (LC/MS) method for the enantiomeric determination of DOPA in dietary supplements containing Mucuna pruriens. L- and D-DOPA were ultrasonically extracted with $1 \%$ formic acid aqueous solution. The isolated extracts were analyzed by LC/MS using a Crownpak CR $(-)$ column at $30^{\circ} \mathrm{C}$. The mass spectrometer was operated in the positive mode of electrospray ionization, and the mobile phase was aqueous formic acid ( $\mathrm{pH} 2.0)$. L-DOPA-ring- $d_{3}$ was used as an internal standard. The method was validated for a dietary supplement spiked with L- and D-DOPA at 50 and $500 \mu \mathrm{g} / \mathrm{g}$, respectively, and the recoveries of the DOPA enantiomers were between $97.5 \%$ and $101.3 \%$. Relative standard deviation values of repeatability and intermediate precision were less than 7\%. The method was applied to 14 dietary supplements. L-DOPA was detected in these supplements in the range of $0.88-12.8 \mathrm{mg} / \mathrm{unit}$. D-DOPA was not detected.
\end{abstract}

Key words: chiral separation; DOPA; Mucuna pruriens; dietary supplement; LC/MS

\section{Introduction}

Mucuna pruriens (commonly known as cowhage, velvet beans, and hassho-mame in Japan) is a climbing legume endemic to India and other tropical regions such as Central and South America. M. pruriens seeds are useful for the treatment of male infertility and nervous disorders and are used as an aphrodisiac according to Ayurvedic medicine. A powder formulation of $M$. pruriens seeds is used for the treatment of Parkinson's disease ${ }^{1)}$. These seeds contain 3-6\% 3,4-dihydroxy-L-phenylalanine (L-DOPA, levodopa ${ }^{2)}$, a pharmaceutical compound used for the treatment of Parkinson's disease. D-DOPA, the enantiomer of L-DOPA, is therapeutically ineffective and has adverse effects such as granulocytopenia ${ }^{3), 4)}$ (Fig. 1).

In recent years, with the increase in health consciousness among individuals, the consumption of dietary supplements has increased. Consequently, many cases of harmful health effects due to dietary supplements have<smiles>N[C@H](Cc1ccc(O)c(O)c1)C(=O)O</smiles>

L-DOPA<smiles>N[C@H](Cc1ccc(O)c(O)c1)C(=O)O</smiles>

D-DOPA

Fig. 1. Structures of DOPA enantiomers

\footnotetext{
* t.hsgw20@pref.chiba.lg.jp
}

been reported ${ }^{5)} . M$. pruriens is used as an ingredient of dietary supplements that appear to have a stimulating effect. Since L-DOPA is contained in $M$. pruriens, there may be health risks associated with the intake of dietary supplements containing $M$. pruriens.

It has been shown that some ingredients of some health food products (dietary supplements) do not originate from labeled materials ${ }^{6), 7)}$. It was also reported that compounds used as indicators for ingredients of labeled materials were not detected in some health food products $^{* 1}$. Moreover, the presence of synthetic drugs intentionally added to some health food products has been reported $^{8-10)}$. Because ingredients of some dietary supplements are different from the materials indicated on the package, it is possible that chemically synthesized DOPA may be used as an ingredient of dietary supplements instead of $M$. pruriens. If D-DOPA or racemic DOPA was used, D-DOPA would be present in these products.

We have developed an HPLC method for the determination of L-DOPA in dietary supplements containing $M$. pruriens using a hydrophilic interaction chromatography column, as described in our previous report ${ }^{11)}$. However, L- and D-DOPA were not separated by this method. Since the expected biological activity is exhibited by L-DOPA, but not D-DOPA, it is important to carry

*1 Ohnuma, M., Kobayashi, S., Hakamatsuka, T., Goda, Y. Abstracts of papers, 13th Annual Meeting of Japanese Society of Food Chemistry, Tokyo, 2007, p. 44. 
out an enantiomeric determination to differentiate them. However, an enantiomeric determination of DOPA in dietary supplements has not been reported to date.

Several analytical HPLC-UV methods have been developed to determine L- and D-DOPA ${ }^{12), 13)}$. However, the sensitivity and specificity of these methods are insufficient for the determination of these compounds in dietary supplements. In this study, we established a simple and rapid chiral liquid chromatography/mass spectrometry (LC/MS) method for the enantiomeric determination of DOPA in dietary supplements containing $M$. pruriens and applied this method to commercial dietary supplements.

\section{Materials and Methods}

\section{Sample}

Fourteen dietary supplements were purchased over the internet. According to the labels, these products (tablets, capsules, and softgels) contained $M$. pruriens. $M$. pruriens seeds (stock No. 55132) were obtained from the Genebank of the National Institute of Agrobiological Sciences. A dietary supplement (tablet) that did not contain DOPA enantiomers was purchased from a drugstore for method validation.

\section{Standard and reagents}

L-DOPA was purchased from Alfa Aesar (Ward Hill, MA, USA). D-DOPA, L-DOPA-ring- $d_{3}$, and LC/MS grade acetonitrile were purchased from Sigma-Aldrich (St. Louis, MO, USA). All other reagents (reagent grade) were purchased from Wako Pure Chemical Industries, Ltd. (Osaka, Japan).

\section{Standard solutions}

Stock standard solutions were prepared by accurately weighing $10 \mathrm{mg}$ of each compound into separate $10-\mathrm{mL}$ volumetric flasks, then dissolving and diluting to the specific volume with $1 \%$ formic acid aqueous solution. A mixed standard solution was prepared by diluting $1 \mathrm{~mL}$ of each stock standard solution to $10 \mathrm{~mL}$ in a volumetric flask with the $1 \%$ formic acid aqueous solution. Calibration standard solutions were prepared in the concentration range $0.05-100 \mu \mathrm{g} / \mathrm{mL}$. An internal standard solution was prepared by weighing $10 \mathrm{mg}$ of L-DOPA-ring- $d_{3}$ into a $100-\mathrm{mL}$ volumetric flask, then dissolving and diluting to the specific volume with $1 \%$ formic acid aqueous solution. The internal standard solution $(100 \mu \mathrm{L})$ was spiked into $1 \mathrm{~mL}$ of all calibration standard solutions.

\section{$L C / M S$ analysis}

The LC/MS system consisted of an Alliance 2695 separation module (Waters, Milford, MA, USA) and a ZQ mass spectrometer (Waters). Chromatographic separation was achieved using a Crownpak CR (-) (150 mm $\times$ $4.0 \mathrm{~mm}$ i.d.; $5 \mu \mathrm{m}$; Daicel, Osaka, Japan). The mobile phase was aqueous formic acid $(\mathrm{pH} 2.0)$. The flow rate of the mobile phase was $0.25 \mathrm{~mL} / \mathrm{min}$, and the injection volume was $5 \mu \mathrm{L}$. The column temperature was maintained at $30^{\circ} \mathrm{C}$. The mass spectrometer was operated in the positive mode of electrospray ionization (ESI). The instrument parameters were as follows: source temperature, $120^{\circ} \mathrm{C}$; desolvation temperature, $350^{\circ} \mathrm{C}$; capillary voltage, $3 \mathrm{kV}$; cone voltage, 20 and $40 \mathrm{~V}$; and desolvation gas (nitrogen) flow, $500 \mathrm{~L} / \mathrm{hr}$. Mass analysis was performed in the selected ion monitoring (SIM) mode. Table 1 shows the monitor ion and optimized cone voltage to confirm and quantify L- and D-DOPA.

\section{Sample preparation}

The tablets and the contents of the capsules were finely powdered using a grinder, and the softgel was carefully opened. Fifty milligrams of each of these samples was transferred into individual test tubes, and $10 \mathrm{~mL}$ of $1 \%$ formic acid aqueous solution was added. This mixture was ultrasonically extracted for $15 \mathrm{~min}$. After centrifuging at $1,300 \times g$ for $10 \mathrm{~min}$, the supernatant was transferred to a $25-\mathrm{mL}$ volumetric flask. The precipitate was re-extracted with $10 \mathrm{~mL}$ of $1 \%$ formic acid aqueous solution under the same conditions and centrifuged. The supernatants collected during the extractions were combined, and the volume was adjusted to $25 \mathrm{~mL}$ with $1 \%$ formic acid aqueous solution. A portion of this solution was filtered through a 0.45- $\mu \mathrm{m}$ polytetrafluoroethylene membrane filter (Millipore, Billerica, MA, USA). The internal standard solution $(100 \mu \mathrm{L})$ was spiked into $1 \mathrm{~mL}$ of the filtrate.

\section{Results and Discussion}

\section{LC/MS analysis}

Cellulose-type columns (Chiralpak IB, Chiralpak IC, Chiralpak OD-RH, Chiralpak OJ-RH, and Chiralpak OZ-RH) and amylase-type columns (Chiralpak IA, Chiralpak AD-RH, Chiralpak AS-RH, and Chiralpak AY-RH) were not able to separate DOPA enantiomers. Thus, Crownpak CR $(+)$ and Crownpak CR (-), which are suitable for chiral separation of amino acids ${ }^{14)}$, were tested. DOPA enantiomers were completely separated using these columns. Since the peak shapes of DOPA enantiomers on the Crownpak CR $(-)$ were better than those on the Crownpak CR $(+)$, the Crownpak CR (-) was used in this study.

To increase the sensitivity and specificity for the DOPA enantiomers, a mass spectrometer was used as the detector. Aqueous formic acid ( $\mathrm{pH}$ 2.0) and aqueous formic acid ( $\mathrm{pH} 2.0)$-methanol (19:1) were tested as the mobile phase. The separation of L- and D-DOPA was insufficient with aqueous formic acid ( $\mathrm{pH}$ 2.0)-methanol $(19: 1)$ (data not shown). Thus, aqueous formic acid

Table 1. SIM settings for MS analysis

\begin{tabular}{cccc}
\hline \hline Compound & $\begin{array}{c}\text { Monitor ion } \\
(\mathrm{m} / \mathrm{z})\end{array}$ & $\begin{array}{c}\text { Cone voltage } \\
(\mathrm{V})\end{array}$ & \\
\hline \multirow{2}{*}{ L-DOPA } & 198 & 20 & Target ion \\
& 152 & 40 & Qualifier ion \\
D-DOPA & 198 & 20 & Target ion \\
& 152 & 40 & Qualifier ion \\
L-DOPA-ring- $d_{3}$ & 201 & 20 & Target ion \\
& 155 & 40 & Qualifier ion \\
\hline
\end{tabular}


( $\mathrm{pH}$ 2.0) was used as the mobile phase.

The effect of the column oven temperature $\left(25-35^{\circ} \mathrm{C}\right)$ on the resolution of the DOPA enantiomers was investigated. The resolution factor $\left(R_{\mathrm{s}}\right)$ of L- and D-DOPA decreased with increasing temperature; $R_{\mathrm{s}}$ at column temperatures of $25^{\circ} \mathrm{C}, 30^{\circ} \mathrm{C}$, and $35^{\circ} \mathrm{C}$ were $2.5,1.9$, and 1.4 , respectively (Fig. 2). Considering the temperature stability of the column oven and the resolution of the DOPA enantiomers, the oven temperature was set at $30^{\circ} \mathrm{C}$.

DOPA enantiomers responded well to ESI in the positive mode. Protonated molecules $[\mathrm{M}+\mathrm{H}]^{+}(\mathrm{m} / \mathrm{z} 198)$ and fragment ions $(\mathrm{m} / z$ 152, representing the loss of $\mathrm{HCOOH})$ of these enantiomers were obtained at a cone voltage of 20 and $40 \mathrm{~V}$, respectively. Figure $3 \mathrm{~A}$ shows the SIM chromatogram of the standard solution of $\mathrm{L}^{-}$and D-DOPA. To minimize matrix effects, such as ion suppression/enhancement and change of retention time, a known amount of the L-DOPA-ring- $d_{3}$ was added as an internal standard.

\section{Evaluation of sample preparation}

In our previous report, acetonitrile-water-formic acid (50:50:1) was used as an extraction solvent for L-DOPA in dietary supplements containing $M$. pruriens $^{11)}$. However, it was expected that acetonitrile-water-formic acid (50:50:1) would be unsuitable as an extraction solvent for the chiral LC/MS analysis because of the presence of

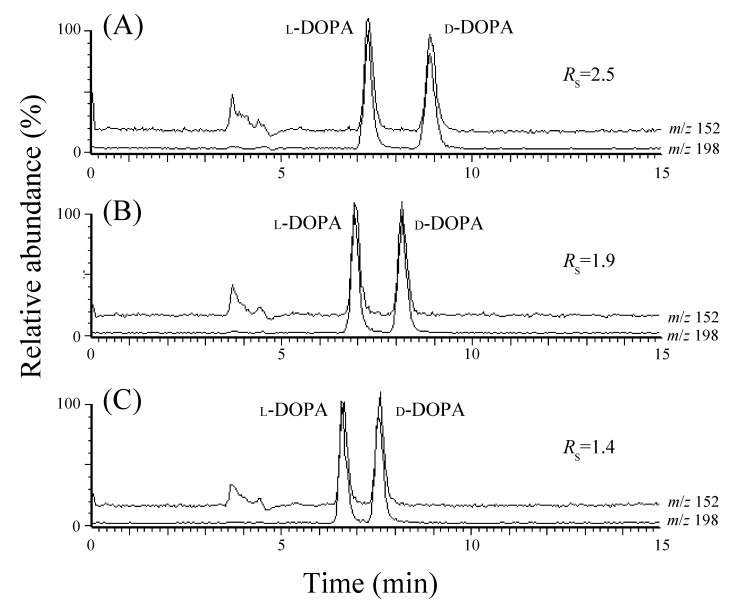

Fig. 2. Effect of column oven temperature on chromatographic separation of DOPA enantiomers $(1 \mu \mathrm{g} / \mathrm{mL}$, each)

(A) $25^{\circ} \mathrm{C}$, (B) $30^{\circ} \mathrm{C}$, and (C) $35^{\circ} \mathrm{C}$ an organic solvent in the extraction solvent. $\mathrm{L}^{-}$and D-DOPA are soluble in water and dilute formic acid ${ }^{15)}$. Therefore, we used 1\% formic acid aqueous solution as an extraction solvent and investigated the appropriate number of extraction steps. $M$. pruriens seed powder (50 mg) was transferred into a test tube and $10 \mathrm{~mL}$ of $1 \%$ formic acid aqueous solution was added. The mixtures were ultrasonically extracted for $15 \mathrm{~min}$. The supernatants were isolated by centrifugation $(1,300 \times g$ for $10 \mathrm{~min})$. The precipitates were re-extracted twice with $10 \mathrm{~mL}$ of the $1 \%$ formic acid aqueous solution and centrifuged. The supernatants obtained from each extraction were filtered, and $100 \mu \mathrm{L}$ of the internal standard solution was spiked into $1 \mathrm{~mL}$ of the filtrate. The resulting solutions were analyzed by LC/MS. As shown in Table 2, minimal L-DOPA was obtained in the third extraction, and D-DOPA was not detected in any extraction. Since L-DOPA was extracted to the extent of greater than 99.9\% after the second extraction, two ultrasonic extractions were applied in further analyses.

Calibration curve, limit of detection, limit of quantification and precision

The linearity, limit of detection, and limit of quantification were determined and are presented in Table 3. Calibration curves of the DOPA enantiomers showed

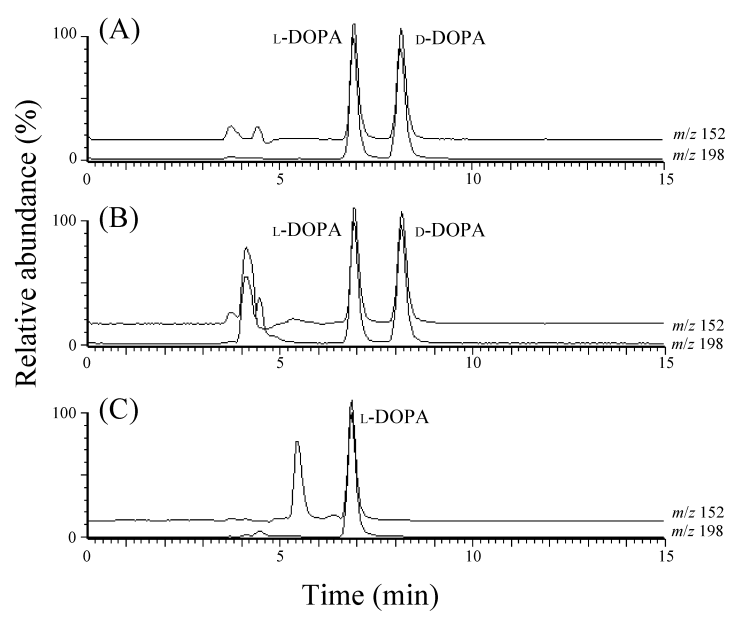

Fig. 3. SIM chromatograms of (A) standard solution of DOPA enantiomers ( $1 \mu \mathrm{g} / \mathrm{mL}$ of each), (B) extract of sample spiked with $50 \mu \mathrm{g} / \mathrm{g}$ of each, and (C) typical sample extract (sample No. 6)

Table 2. Effect of the number of extraction steps in repetitive extraction of M. pruriens seeds

\begin{tabular}{ccccc}
\hline \hline & \multicolumn{2}{c}{ L-DOPA } & & D-DOPA \\
\cline { 2 - 3 } & Concentration $^{\mathrm{a}}(\mu \mathrm{g} / \mathrm{mL})$ & Extraction rate $\left.^{2} \%\right)$ & & Concentration $^{\mathrm{a}}(\mu \mathrm{g} / \mathrm{mL})$ \\
\hline First extraction & $283 \pm 11.6$ & 97.78 & $\mathrm{ND}^{\mathrm{b}}$ \\
Second extraction & $6.23 \pm 1.03$ & 2.17 & $\mathrm{ND}$ & $\mathrm{ND}$ \\
Third extraction & $0.17 \pm 0.03$ & 0.06 & \\
\hline Total & & 100.0 & \\
\hline
\end{tabular}

\footnotetext{
${ }^{\text {a }}$ Values are means \pm standard deviation $(n=3)$

${ }^{\mathrm{b}}$ Not detected
} 
Table 3. Calibration curves, limit of detection, and limit of quantification

\begin{tabular}{cccc}
\hline \hline Analyte & Calibration curve & $r^{2 \mathrm{~b}}$ & $\mathrm{LOD}^{\mathrm{c}}(\mu \mathrm{g} / \mathrm{mL})$ \\
\hline L-DOPA & $y=0.8328 x+0.1658$ & 0.9998 & 0.003 \\
D-DOPA & $y=0.8589 x+0.2197$ & 0.9997 & 0.003 \\
\hline
\end{tabular}

${ }^{a} y$ : peak area of analyte/peak area of internal standard, $x$ : concentration of analyte $(\mu \mathrm{g} / \mathrm{mL})$

${ }^{\mathrm{b}}$ Correlation coefficient

${ }^{\mathrm{c}}$ Limit of detection

${ }^{\mathrm{d}}$ Limit of quantification

Table 4. Recoveries, repeatability, and intermediate precision

\begin{tabular}{cccc}
\hline \hline Analyte & $\begin{array}{c}\text { Spiked amount } \\
(\mu \mathrm{g} / \mathrm{g})\end{array}$ & $\begin{array}{c}\text { Recovery } \\
(\%)\end{array}$ & \multicolumn{2}{c}{$\begin{array}{c}\text { Repeatability } \\
(\%)\end{array}$} \\
\hline & 50 & 101.3 & 3.40 \\
L-DOPA & 500 & 100.5 & 2.78 \\
D-DOPA & 50 & 97.5 & 6.34 \\
& 500 & 98.5 & 3.69 \\
\hline
\end{tabular}

${ }^{a}$ Means of 10 replicates

Table 5. Contents of L- and D-DOPA in dietary supplements containing $M$. pruriens

\begin{tabular}{|c|c|c|c|c|c|c|}
\hline Sample No. & Form & $\begin{array}{l}\text { L-DOPA } \\
\text { content }^{\mathrm{a}} \\
\text { (mg/unit) }\end{array}$ & $\begin{array}{l}\text { D-DOPA } \\
\text { content } \\
\text { (mg/unit) }\end{array}$ & $\begin{array}{l}\text { Average weight } \\
\text { (mg/unit) }\end{array}$ & $\begin{array}{c}\text { Indicated } \\
\text { maximum dosage } \\
\text { (unit/day) }\end{array}$ & $\begin{array}{c}\text { Calculated maximum } \\
\text { intake of } \mathrm{L}-\mathrm{DOPA}^{\mathrm{c}} \\
(\mathrm{mg} / \text { day })\end{array}$ \\
\hline 1 & Capsule & 4.31 & $\mathrm{ND}^{\mathrm{b}}$ & 301 & 5 & 21.6 \\
\hline 2 & Tablet & 6.84 & ND & 501 & 4 & 27.4 \\
\hline 3 & Softgel & 0.88 & ND & 371 & 3 & 2.64 \\
\hline 4 & Tablet & 9.17 & ND & 252 & 8 & 73.4 \\
\hline 5 & Tablet & 12.8 & ND & 308 & 6 & 76.8 \\
\hline 6 & Tablet & 1.33 & ND & 254 & 8 & 10.6 \\
\hline 7 & Tablet & 4.16 & ND & 254 & 6 & 25.0 \\
\hline 8 & Capsule & 3.60 & ND & 349 & 5 & 18.0 \\
\hline 9 & Tablet & 0.92 & ND & 255 & 6 & 5.52 \\
\hline 10 & Tablet & 3.90 & ND & 355 & 2 & 7.80 \\
\hline 11 & Tablet & 1.82 & $\mathrm{ND}$ & 251 & 8 & 14.6 \\
\hline 12 & Capsule & 7.48 & $\mathrm{ND}$ & 306 & 6 & 44.9 \\
\hline 13 & Capsule & 3.76 & ND & 312 & 2 & 7.52 \\
\hline 14 & Capsule & 7.43 & ND & 350 & 2 & 14.9 \\
\hline
\end{tabular}

${ }^{\text {a }}$ Values are means $(n=3)$

b Not detected

good linearity in the range of $0.05-100 \mu \mathrm{g} / \mathrm{mL}$, and the correlation coefficients $\left(r^{2}\right)$ determined for each compound were higher than 0.999 . The limit of detection of each compound was $0.003 \mu \mathrm{g} / \mathrm{mL}(S / N \geq 3)$, and the limit of quantification of each compound was $0.010 \mu \mathrm{g} / \mathrm{mL}$ $(S / N=10)$. This quantification method was validated according to the Japanese method validation guideline ${ }^{* 2}$. A dietary supplement (tablet) that did not contain DOPA enantiomers was spiked with a known amount of standard L- and D-DOPA (50 and $500 \mu \mathrm{g} / \mathrm{g}$, each) and analyzed in duplicate on five different days. The SIM chromatogram of a spiked sample is shown in Fig. 3B. All recoveries were between 97.5 and $101.3 \%$ (Table 4).

*2 Director Notice of Department of Food Safety, Ministry of Health, Labour and Welfare of Japan, Syoku-An No. 1115001 (Nov. 15, 2007)
Relative standard deviation (RSD) values of the repeatability ranged from $2.78 \%$ to $6.34 \%$ and those of the intermediate precision ranged from $2.96 \%$ to $6.90 \%$. These results suggest that good accuracy and precision can be obtained using this method.

Determination of the content of DOPA enantiomers in commercial dietary supplements

We applied this method to 14 commercial dietary supplements. The SIM chromatogram of a typical sample (No. 6) is shown in Fig. 3C. L-DOPA was detected in these dietary supplements in the range of $0.88-12.8 \mathrm{mg} /$ unit. On the other hand, D-DOPA was not detected in any dietary supplement (Table 5). Since D-DOPA was not detected in any product, it was considered that the ingredients of these dietary supplements could be $M$. pruriens extracts rather than chemically synthesized 
DOPA. The maximum intake of L-DOPA per day was calculated from the L-DOPA content and the maximum dosage indicated on the package of each product. The maximum intake of L-DOPA was found to be in the range of $2.64-76.8 \mathrm{mg} / \mathrm{day}$. The initial clinical dosage of L-DOPA is $0.2-0.6 \mathrm{~g} /$ day divided over 1,2 , or 3 doses $^{16)}$. As per the dosage mentioned on the package, if an individual took 6 tablets of sample No. 5, the amount of L-DOPA ingested would be approximately one-third of the minimum clinical L-DOPA dosage. Since sensitivity to drugs may vary for each individual depending on the person's disease state, age, weight, and gender, there could be health risks associated with the intake of dietary supplements containing $M$. pruriens.

\section{Acknowledgments}

The authors thank Daicel Corporation for technical assistance on column selection.

\section{References}

1) Katzenschlager, R., Evans, A., Manson, A., Patsalos, P. N., Ratnaraj, N., Watt, H., Timmenrmann, Van der Giessen, R., Lees, A. J. Mucuna pruriens in Parkinson's disease: a double blind clinical and pharmacological study. J. Neurol. Neurosurg. Psychiatry, 75, 1672-1677 (2004).

2) Jellin, J. M., Batz, F., Hitchens, K. (Yamada, K. et al. trs.) Natural Medicines Comprehensive Database. (Kenkoshokuhin Detabesu.) Tokyo, Daiichi Shuppan, 2001, p. 160-162 (ISBN 978-4-8041-1096-7)

3) Cotzias, G. C., Papavasiliou, P. S., Gellene, R. Modification of Parkinsonism-chronic treatment with L-DOPA. N. Engl. J. Med., 280, 337-345 (1969).

4) Barbeau, A. L-DOPA therapy in Parkinson's disease: a critical review of nine years' experience. Can. Med. Assoc. J., 101, 59-68 (1969).

5) Naito, Y. Kenkoshokuhin Chudokuhyakka. Tokyo, Maruzen, 2007, 316 p. (ISBN 978-4-621-0784-2)

6) Sakai, S., Otake, E., Toida, T., Goda, Y. Identification of the origin of chondroitin sulfate in "health foods". Chem. Pharm. Bull., 55, 299-303 (2007).

7) Goda, Y. The safety of health foods and importance of their origin. Yakugaku Zasshi (Journal of the Pharmaceutical Society of Japan), 128, 837-838 (2008).

8) Moriyasu, T., Shigeoka, S., Kishimoto, K., Ishikawa, F., Nakajima, J., Kamimura, H., Yasuda, I. Identification system for sildenafil in health foods. Yakugaku Zasshi, 121, 765-769 (2001).

9) Kumasaka, K., Kojima, T., Doi, K., Satoh, S. Analysis of the oral hypoglycemic agent, glibenclamide, in a health food. Yakugaku Zasshi, 123, 136-141 (2003).

10) Hasegawa, T., Ishii, T., Miyamoto, F., Ito, K. Medicinal components detected in Chinese dietary supplement caused health damages. Chibaken Eiseikenkyusho Kenkyuhokoku (Bulletin of the Chiba Prefectural Institute of Public Health), 29, 37-40 (2005).

11) Hasegawa, T., Ishii T., Takahashi, K., Saijo, M., Fukiwake, T., Nagata, T., Motoki, Y. Quantitative determination of L-DOPA in dietary supplements containing Mucuna pruriens by high performance liquid chromatography. Chibaken Eiseikenkyusho Nenpo (Annual Report of the Chiba Prefectural Institute of Public Health), 60, 53-56 (2011).

12) Gelber, L. R., Neumeyer, J. L. Determination of the enantiomeric purity of levodopa, methyldopa, carbidopa and tryptophan by use of chiral mobile phase high-performance liquid chromatography. J. Chromatogr., 257, 317-326 (1983).

13) Doležalová, M., Tkaczyková, M. Direct high-performance liquid chromatographic determination of the enantiomeric purity of levodopa and methyldopa: comparison with pharmacopoeial polarimetric methods. J. Pharm. Biomed. Anal., 19, 555-567 (1999).

14) Takatori, K., Toyama, S., Fujii, S., Kajiwara, M. Analysis of amino acids by high-performance liquid chromatography with circular dichroism detection. Chem. Pharm. Bull., 43, 1797-1799 (1995).

15) O’Neil, M. J., Smith, A., Heckelman, P. E. eds. The Merck Index, 14th ed. New Jersey, USA, Merck Research Laboratories, 2006, p. 946-947. (ISBN 978-0-9119-1000-1)

16) Drugs in Japan Ethical Drugs 2012, Jiho, Tokyo, 2011, p. 3124-3126. (ISBN 978-4-8407-4202-3) 